\title{
An Operational Semantics for True Concurrency in BDI Agent Systems
}

\author{
Lavindra de Silva \\ Department of Engineering \\ University of Cambridge, UK \\ Lavindra.deSilva@eng.cam.ac.uk
}

\begin{abstract}
Agent programming languages have proved useful for formally modelling implemented systems such as PRS and JACK, and for reasoning about their behaviour. Over the past decades, many agent programming languages and extensions have been developed. A key feature in some of them is their support for the specification of 'concurrent' actions and programs. However, their notion of concurrency is still limited, as it amounts to a nondeterministic choice between (sequential) action interleavings. Thus, the notion does not represent 'true concurrency', which can more naturally exploit multicore computers and multi-robot manufacturing cells. This paper provides a true concurrency operational semantics for a BDI agent programming language, allowing actions to overlap in execution. We prove key properties of the semantics, relating to true concurrency and to its link with interleaving.
\end{abstract}

\section{Introduction}

Agent programming languages have proved useful for formally modelling implemented systems such as Jason (Bordini and Hübner 2010), PRS (Georgeff and Ingrand 1989), and JACK (Busetta et al. 1999), and for reasoning about their behaviour. Over the past decades, many agent programming languages and extensions have emerged, e.g. (Rao 1996; Hindriks et al. 2001; Dastani 2008; Winikoff et al. 2002; Morley and Myers 2004; Sardina, de Silva, and Padgham 2006; Chaouche et al. 2014; de Silva, Meneguzzi, and Logan 2018; de Giacomo, Lespérance, and Levesque 2000). A key feature in some of them is their support for the specification of 'concurrent' actions and programs. However, while their notion of concurrency is useful in some applications, it is still limited as it amounts to a nondeterministic choice between (sequential) action interleavings. Thus, the notion does not represent 'true concurrency', which can more naturally exploit multi-core computers and multi-robot manufacturing cells, e.g. a cell comprising two robot arms that work on a part simultaneously. This paper provides a true concurrency operational semantics for a BDI agent programming language, allowing actions to overlap in execution.

From the implemented BDI agent systems, there are some that support some form of true concurrency, e.g. SPARK (Morley and Myers 2004), JAM (Huber 1999), BDI4JADE

Copyright (C) 2020, Association for the Advancement of Artificial Intelligence (www.aaai.org). All rights reserved.
(Nunes, Lucena, and Luck 2011), PRS, and JACK. ${ }^{1}$ JACK gives four (programmer selectable) definitions for the success of a concurrent program: (1) it immediately succeeds on the successful termination of a branch; (2) it allows all branches to terminate but only succeeds if at least one of them succeeds; (3) it succeeds iff all branches terminate successfully, and immediately fails on the failure of a branch; or (4) it allows all branches to terminate but only succeeds if all branches succeed. We follow this last definition.

There is also related work in concurrent distributed systems, particularly three strands of work that define behaviour in terms of transitions between configurations, using a structural operational semantics (Plotkin 1981). In the first strand (Boudol and Castellani 1988b; 1989), every transition is labelled with a representation of its 'proof', i.e., information comprising the inference rules that were used when deriving the transition. This information enables the extraction of a transition labelled with a partially ordered multiset (pomset) of actions, representing the sequential and concurrent actions performed. A similar transition is extracted in (Degano, De Nicola, and Montanari 1985; 1990), but from information stored in configurations rather than transition labels. In the third strand (Boudol and Castellani 1987; 1988a), transitions are labelled with 'composite actions', which represent pomsets. This avoids the need to extract a pomset-labelled transition 'a posteriori' from a sequence of transitions, as done above.

Our work was inspired by the last two strands: our transitions represent composite actions, which are stored in configurations. However, unlike the above strands, we account for concerns specific to agent programming languages, e.g. goal refinement, plan failure, and constructs beyond actions. Our proposal, called Concurrent CAN (CCAN), is based on the work in (Sardina and Padgham 2011), which refines and extends the CAN agent programming language (Winikoff et al. 2002). We discuss the syntax of CCAN (sec. 2) and its semantics (secs. 3 and 4), and we prove key properties of CCAN, in relation to true concurrency and to its link with interleaving (sec. 5); e.g., we show that if a concurrent program's branches are interleaved as in CAN, Jason, etc., any resulting behaviour can also be produced by the concurrent

\footnotetext{
${ }^{1}$ There are also implemented BDI systems that do not support true concurrency but support action interleaving, e.g. Jason.
} 
program. We then conclude and discuss future work (sec. 6).

\section{CCAN Syntax}

In this paper, we use a first-order language with a vocabulary comprising mutually disjoint and infinite sets of variable, function, predicate, event-goal, and action symbols.

Like (Sardina and Padgham 2011), we define a CCAN agent by a plan-library $\Pi$, an action-library $\Lambda$, and a belief base $\mathcal{B}$. A belief base is a set of ground atoms, and an $\underline{a c-}$ tion-library is a set of action-rules representing actions the agent can perform. An action, denoted by $a$, is of the form $\operatorname{act}(\vec{t})$, where act is an n-ary action symbol representing a function that may affect the external environment, and $\vec{t}=$ $t_{1}, \ldots, t_{n}$ is a list of (possibly ground) terms. An action$\underline{\text { rule, }}$, as in STRIPS, is of the form $\operatorname{act}(\vec{v}): \psi \leftarrow \Phi^{+} ; \Phi^{-}$, where $\vec{v}=v_{1}, \ldots, v_{n}$ is a list of distinct variables; $\psi$, the precondition, is a formula; and $\Phi^{+}$and $\Phi^{-}$, respectively the add-list and delete-list, are each a set of atoms representing the action's effects. Any variable occurring in $\psi, \Phi^{+}$, or $\Phi^{-}$ also occurs in $\vec{v}$. For any action $a$ that occurs in $\Pi$, there is exactly one action-rule $a^{\prime}: \psi \leftarrow \Phi^{+} ; \Phi^{-} \in \Lambda$ such that $a=a^{\prime} \theta$ for some substitution $\theta$; we define $\operatorname{pre}(a, \Lambda)=\psi \theta$ and eff $(a, \Lambda)=\left\langle\Phi^{+} \theta, \Phi^{-} \theta\right\rangle$.

A plan-library $\Pi$ is a set of plan-rules of the form $e v(\vec{t}): \psi \leftarrow P_{b}$, where $e v(\vec{t})$, denoted by $e$, is an eventgoal and $e v$ is an n-ary event-goal symbol; $\psi$, the $\underline{\text { context }}$ condition, is a formula; and $P_{b}$, the plan-body is a "standard operating procedure' for achieving $e$ when $\psi$ holds in $\mathcal{B}$. Formally, a plan-body is a formula in the language defined by the grammar $P_{b}::=$

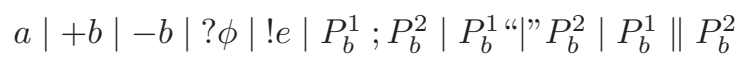

where $+b$ is a belief addition, which adds the atom $b$ to $\mathcal{B} ;-b$ is a belief removal, which removes $b$ from $\mathcal{B} ; ? \phi$ is a test condition, which tests whether formula $\phi$ holds in $\mathcal{B}$; !e is an event-goal program, which states that $e$ needs to be achieved; and $P_{b}^{1} ; P_{b}^{2}$ is a sequential program, which states that $P_{b}^{1}$ must be executed before $P_{b}^{2}$. Finally, $P_{b}^{1} \mid P_{b}^{2}$ is an $\underline{i n-}$ terleaved program, which allows the resulting actions to be interleaved (but not overlapped); ${ }^{2}$ and $P_{b}^{1} \| P_{b}^{2}$ is a (truly) concurrent program, which allows the resulting actions to be interleaved and/or overlapped, as described in (Allen 1983). In the sequel, we use the terms 'concurrency' and 'concurrent' only when referring to the latter type of program or its execution.

We impose two constraints relating to concurrency. First, we limit how a concurrent program $P_{b}^{1} \| P_{b}^{2}$ is interleaved with another program: no other (non-concurrent) step is executed during $P_{b}^{1} \| P_{b}^{2}$; for example, executing interleaved program $\left(a_{1} \| a_{2}\right) \mid a_{3}$ will result in action $a_{3}$ happening either before or after both $a_{1}$ and $a_{2}$. Second, no two branches of a concurrent program are 'related'. Two branches are related if $(i)$ a variable appearing in one branch can be bound by the other, or (ii) the same atom can be both asserted by one branch and checked or asserted by the other.

\footnotetext{
${ }^{2}$ The exact schedule that emerges from the resulting actions will be based on runtime choices.
}

To formalise the assumption that the branches of a concurrent program are unrelated, we define some auxiliary notions. Let $\Pi$ and $\Lambda$ be a plan- and an action-library, respectively. First, given any expression $E$, we use $\operatorname{ATS}(E)$ to denote the set of atoms occurring in $E$. Second, given an eventgoal $e$, we use $\operatorname{REL}(e, \Pi)=\left\{\psi \theta: P_{b} \theta \mid e^{\prime}: \psi \leftarrow P_{b} \in\right.$ $\left.\Pi, \theta=\operatorname{mgu}\left(e, e^{\prime}\right)\right\}$ to denote the relevant plan-rules for $e$, i.e., rules with 'heads' $e^{\prime}$ that match $e$ via a most general unifier (mgu). Third, given a plan-body $P_{b}$, we recursively define the set of atoms that are possibly checked by $P_{b}$ as follows: $\operatorname{CHK}\left(P_{b}, \Pi, \Lambda\right)=$

$$
\begin{cases}\emptyset & \text { if } P_{b} \in\{+b,-b\}, \\ \operatorname{ATS}(\phi) & \text { if } P_{b}=? \phi, \\ \operatorname{ATS}(p r e(a, \Lambda)) & \text { if } P_{b}=a, \\ \bigcup_{\psi: P_{b}^{\prime} \in \operatorname{ReL}(e, \Pi)} \operatorname{ATS}(\psi) \cup \operatorname{CHK}\left(P_{b}^{\prime}, \Pi, \Lambda\right) & \text { if } P_{b}=! e, \\ \operatorname{CHK}\left(P_{b}^{1}, \Pi, \Lambda\right) \cup \operatorname{CHK}\left(P_{b}^{2}, \Pi, \Lambda\right) & \text { if } P_{b} \in\left\{P_{b}^{1} \mid P_{b}^{2},\right. \\ & \left.P_{b}^{1} \| P_{b}^{2}, P_{b}^{1} ; P_{b}^{2}\right\} .\end{cases}
$$

Similarly, we define the set of atoms that are possibly asserted by $P_{b}$ as follows: $\operatorname{ASS}\left(P_{b}, \Pi, \Lambda\right)=$

$$
\begin{cases}\{b\} & \text { if } P_{b} \in\{+b,-b\} \\ \emptyset & \text { if } P_{b}=? \phi \\ \operatorname{ATS}(e f f(a, \Lambda)) & \text { if } P_{b}=a, \\ \bigcup_{\psi: P_{b}^{\prime} \in \operatorname{ReL}(e, \Pi)} \operatorname{ASS}\left(P_{b}^{\prime}, \Pi, \Lambda\right) & \text { if } P_{b}=! e \\ \operatorname{ASS}\left(P_{b}^{1}, \Pi, \Lambda\right) \cup \operatorname{ASS}\left(P_{b}^{2}, \Pi, \Lambda\right) & \text { if } P_{b} \in\left\{P_{b}^{1} \mid P_{b}^{2},\right. \\ & \left.P_{b}^{1} \| P_{b}^{2}, P_{b}^{1} ; P_{b}^{2}\right\}\end{cases}
$$

Finally, for any concurrent program $P_{b}^{1}\|\ldots\| P_{b}^{n}$ occurring in $\Pi$, we assume that for any $i, j \in[1, n]$, with $i \neq j$, there does not exist a $(i)$ variable that occurs in both $P_{b}^{i}$ and $P_{b}^{j}$, and (ii) unifier for any pair of atoms $\left\{l, l^{\prime}\right\}$, where $l \in$ $\operatorname{CHK}\left(P_{b}^{i}, \Pi, \Lambda\right) \cup \operatorname{ASS}\left(P_{b}^{i}, \Pi, \Lambda\right)$ and $l^{\prime} \in \operatorname{ASS}\left(P_{b}^{j}, \Pi, \Lambda\right){ }^{3}$

\section{Single-Intention CCAN Semantics}

We define an agent configuration as a tuple $[\Pi, \Lambda, \mathcal{B}, \mathcal{A}, \Gamma]$, where $\Pi$ is a plan-library; $\Lambda$ is an action-library; $\mathcal{B}$ is a belief base; and $\mathcal{A}$ is an action history representing the sequential and concurrent actions executed so far. Formally, an action $\underline{\text { history }}$ is a formula in the language defined by the grammar

$$
\mathcal{A}::=a\left|\mathcal{A}_{1} ; \mathcal{A}_{2}\right| \mathcal{A}_{1} \| \mathcal{A}_{2}
$$

where $\mathcal{A}_{1} \| \mathcal{A}_{2}$ is an abstract representation for all the associated 'schedules', each comprising interleaved and/or overlapping actions. Finally, $\Gamma$ is a set of programs, or 'intentions', each of which is the current evolution in the execution of a plan-body that is being pursued in order to achieve a top-level event-goal. As it is usual with small-step operational semantics of programming languages, the syntax of plan-bodies has to be extended with new constructs to represent these current evolutions. Formally, a program is a formula in the language defined by the grammar $P::=$

$$
\begin{aligned}
& a|+b|-b|? \phi| ! e\left|P_{1} ; P_{2}\right| P_{1} \text { " " } P_{2}\left|P_{1} \| P_{2}\right| \\
& \left.\eta \mid e: 0\left\{\psi_{1}: P_{1}, \ldots, \psi_{n}: P_{n}\right\}\right)\left|P_{1} \triangleright P_{2}\right| P_{1} \nVdash P_{2}
\end{aligned}
$$

where $\eta$ (or 'nil') indicates that a program has finished, i.e., successfully terminated; $P_{1} \nVdash P_{2}$ indicates that a

${ }^{3}$ We refer the reader to (de Silva, Sardina, and Padgham 2016) for insights into algorithms for checking these conditions. 


$$
\begin{aligned}
& \frac{\Delta=\operatorname{REL}(e) \neq \emptyset}{[\mathcal{B}, \mathcal{A}, ! e] \stackrel{\text { ONE }}{\longrightarrow}[\mathcal{B}, \mathcal{A}, e:(\Delta)]} E v \quad \frac{\psi: P \in \Delta \quad \mathcal{B} \models \psi \theta}{[\mathcal{B}, \mathcal{A}, e:(\Delta)] \stackrel{\text { ONE }}{\longrightarrow}[\mathcal{B}, \mathcal{A}, P \theta \triangleright e:(\Delta \backslash\{\psi: P\})]} S e l \\
& \frac{\left[\mathcal{B}, \mathcal{A}, P_{1}\right] \stackrel{\mathrm{ONE}}{\longrightarrow}\left[\mathcal{B}^{\prime}, \mathcal{A}^{\prime}, P_{1}^{\prime}\right]}{\left[\mathcal{B}, \mathcal{A}, P_{1} ; P_{2}\right] \stackrel{\mathrm{ONE}}{\longrightarrow}\left[\mathcal{B}^{\prime}, \mathcal{A}^{\prime}, P_{1}^{\prime} ; P_{2}\right]} \operatorname{Seq} \frac{\operatorname{FIN}\left(P_{1}\right)}{\left[\mathcal{B}, \mathcal{A}, P_{1} ; P_{2}\right] \stackrel{\mathrm{ONE}}{\longrightarrow}\left[\mathcal{B}, \mathcal{A}, P_{2}\right]} S e q_{2} \\
& \left.\left.\frac{\left[\mathcal{B}, \mathcal{A}, P_{1}\right] \stackrel{\mathrm{ONE}}{\longrightarrow}\left[\mathcal{B}^{\prime}, \mathcal{A}^{\prime}, P_{1}^{\prime}\right]}{\left[\mathcal{B}, \mathcal{A}, P_{1} \mid P_{2}\right] \stackrel{\text { ONE }}{\longrightarrow}\left[\mathcal{B}^{\prime}, \mathcal{A}^{\prime}, P_{1}^{\prime} \mid P_{2}\right]}\right|_{1} \frac{\left[\mathcal{B}, \mathcal{A}, P_{2}\right] \stackrel{\mathrm{ONE}}{\longrightarrow}\left[\mathcal{B}^{\prime}, \mathcal{A}^{\prime}, P_{2}^{\prime}\right]}{\left[\mathcal{B}, \mathcal{A}, P_{1} \mid P_{2}\right] \stackrel{\mathrm{ONE}}{\longrightarrow}\left[\mathcal{B}^{\prime}, \mathcal{A}^{\prime}, P_{1} \mid P_{2}^{\prime}\right]}\right|_{2} \\
& \frac{\left[\mathcal{B}, \mathcal{A}, P_{1}\right] \stackrel{\text { ONE }}{\longrightarrow}\left[\mathcal{B}^{\prime}, \mathcal{A}^{\prime}, P_{1}^{\prime}\right]}{\left[\mathcal{B}, \mathcal{A}, P_{1} \triangleright P_{2}\right] \stackrel{\text { ONE }}{\longrightarrow}\left[\mathcal{B}^{\prime}, \mathcal{A}^{\prime}, P_{1}^{\prime} \triangleright P_{2}\right]} \triangleright_{\text {step }} \quad \frac{\left[\mathcal{B}, \mathcal{A}, P_{1}\right] \stackrel{\text { ONE }}{\longrightarrow} \neg \mathrm{FIN}\left(P_{1}\right)\left[\mathcal{B}, \mathcal{A}, P_{2}\right] \stackrel{\text { ONE }}{\longrightarrow}\left[\mathcal{B}, \mathcal{A}, P_{2}^{\prime}\right]}{\left[\mathcal{B}, \mathcal{A}, P_{1} \triangleright P_{2}\right] \stackrel{\text { ONE }}{\longrightarrow}\left[\mathcal{B}, \mathcal{A}, P_{2}^{\prime}\right]} \triangleright_{\text {fail }} \\
& \frac{\mathcal{B} \models \phi}{[\mathcal{B}, \mathcal{A}, ? \phi] \stackrel{\mathrm{x}}{\longrightarrow}[\mathcal{B}, \mathcal{A}, \eta]} \text { Test } \quad \frac{a^{\prime}: \psi \leftarrow \Phi^{+} ; \Phi^{-} \in \Lambda \quad a^{\prime} \theta=a \quad \mathcal{B} \models \psi \theta}{[\mathcal{B}, \mathcal{A}, a] \stackrel{\mathrm{x}}{\longrightarrow}\left[\left(\mathcal{B} \backslash \Phi^{-} \theta\right) \cup \Phi^{+} \theta, \mathcal{A} ; a, \eta\right]} \text { Act }
\end{aligned}
$$

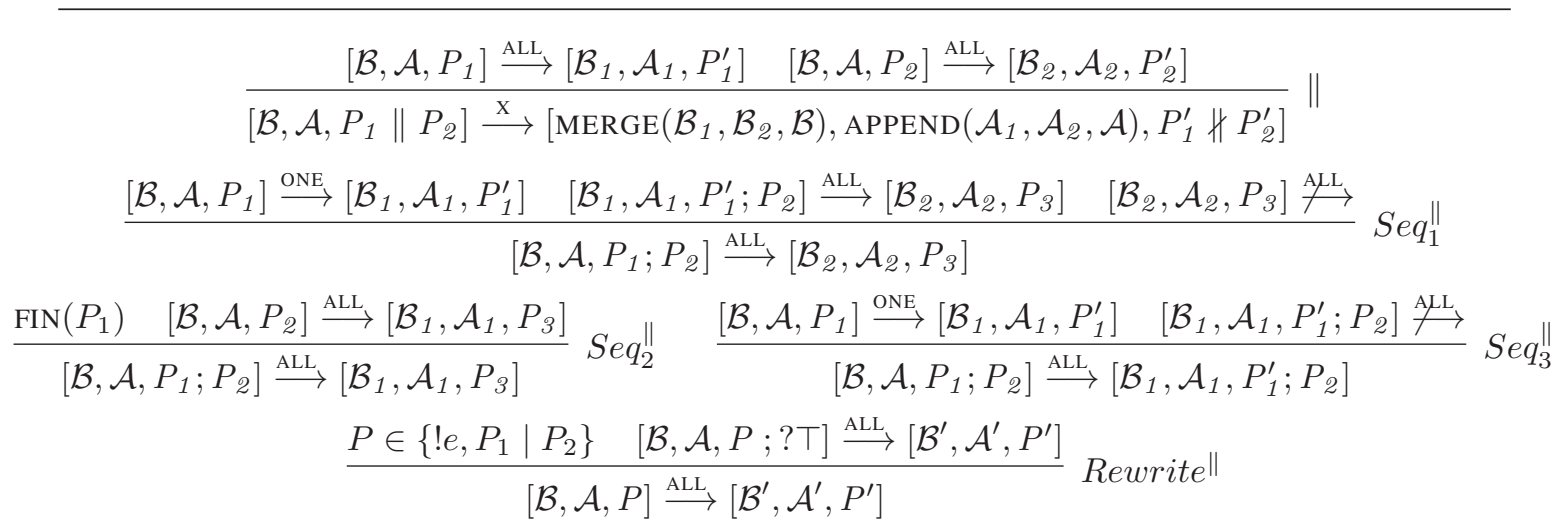

Figure 1: CCAN derivation rules for configurations with single intentions.

concurrent program has terminated (not necessarily successfully); $e:\left(\left\{\psi_{1}: P_{1}, \ldots, \psi_{n}: P_{n}\right\}\right)$ represents the set of plan-rules that are relevant for achieving event-goal $e$; and 'failure handling' program $P \triangleright P^{\prime}$, with $P^{\prime}=e$ : $\left(\left\{\psi_{1}: P_{1}, \ldots, \psi_{n}: P_{n}\right\}\right)$, executes program $P$ in order to achieve event-goal $e$, and if $P$ fails, an alternative program (plan-body) $P_{i}$ is tried if it is applicable. Note that a program is more general than those generated by our semantics.

Like (Sardina and Padgham 2011), we define a transition relation on configurations in terms of a set of derivation rules (Plotkin 1981); we omit the elements $\Pi$ and $\Lambda$ from configurations in our transitions as those elements do not change between transitions. A derivation rule has an antecedent and a conclusion: the latter is a single transition, and the former is either empty or a conjunction of auxiliary conditions and/or transitions representing 'internal' execution steps. In this paper we only use labelled transitions. A transition $C \stackrel{\text { ONE }}{\longrightarrow} C^{\prime}$ indicates that doing one execution step on configuration $C$, which may involve multiple internal execution steps, yields configuration $C^{\prime}$. A transition $C \stackrel{\mathrm{ALL}}{\longrightarrow} C^{\prime}$ indicates that $C^{\prime}$ is a result of doing all possible internal execution steps from $C$. Intuitively, ONE-type transitions model behaviour in the context of standard execution, and ALL-type ones model behaviour in the context of concurrent execution.

We first give our semantics for single-intention configura- tions of the form $[\Pi, \Lambda, \mathcal{B}, \mathcal{A}, P]$, where $P$ is a program. In sec. 3.1 we give derivation rules for standard programs, and in sec. 3.2 we give rules that relate to concurrent programs.

\subsection{Derivation Rules for Standard Programs}

Fig. 1 (top half) shows the derivation rules for standard CCAN programs, including interleaved programs.

Rule $E v$ creates the set $\Delta$ of relevant plan-rules for a given event-goal program !e. Rule $\mathrm{Sel}$ selects an applicable plan-rule for an event-goal $e$ from its relevant plan-rules $\Delta$, and schedules the corresponding plan-body for execution.

Rules $S e q_{1}$ and $S e q_{2}$ give semantics for sequential execution: $S e q_{1}$ executes one step on a sequential program $P_{1} ; P_{2}$ by executing a step on its first program $P_{1}$, and $S e q_{2}$ removes $P_{1}$ if it has finished (as we define in sec. 3.2), e.g., if $P_{1}=\eta$. Rules $\left.\right|_{1}$ and $\left.\right|_{2}$ give semantics for interleaved execution: given an interleaved program $P_{1} \mid P_{2}$, one step is executed either on $P_{1}$ (using rule $\left.\right|_{1}$ ) or on $P_{2}$ (using $\left.\right|_{2}$ ).

Rules $\triangleright_{\text {step }}$ and $\triangleright_{\text {fail }}$ give semantics for executing a previously selected plan-body and for failure handling, respectively: rule $\triangleright_{\text {step }}$ executes one step on a program $P_{1} \triangleright P_{2}$ by executing a step on $P_{1}$, provided it has neither failed nor finished, and rule $\triangleright_{\text {fail }}$ removes $P_{1}$ if it has failed, and executes a step on program $P_{2}=e:(\Delta)$. A program has failed if it has not finished (as in the second condition in the an- 
tecedent of $\triangleright_{\text {fail }}$ ) but it is 'stuck' (as in the first condition in the antecedent of $\left.\nabla_{\text {fail }}\right)$, i.e., it is not possible to execute a step on the program (e.g., an event-goal program !e when there are no relevant plan-rules for $e$ ). Given a configuration $C$, we use $C \stackrel{\text { ONE }}{\longrightarrow}$ as an abbreviation for $\exists C^{\prime}, C \stackrel{\text { ONE }}{\longrightarrow} C^{\prime}$ (and use $C \stackrel{\mathrm{ALL}}{\longrightarrow}, C \stackrel{\mathrm{ONE}}{\longrightarrow}$, and $C \stackrel{\mathrm{ALL}}{\longrightarrow}$ similarly).

Finally, rule Test executes one step on a test program $? \phi$ if condition $\phi$ holds in the belief base, and rule Act gives semantics for actions. This rule's antecedent checks whether the relevant action-rule of a given action $a$ is applicable, and the conclusion applies the action's effects to the belief base, and appends $a$ to action history $\mathcal{A}$. When the transition label $\mathrm{X}$ occurs in a derivation rule, the associated transition represents both transition types (ONE and ALL).

\subsection{Derivation Rules for Concurrent Programs}

Fig. 1 (bottom half) shows the derivation rules that relate to concurrent programs. The main rule $\|$ executes one step on a program $P_{1} \| P_{2}$, which amounts to independently doing all possible internal execution steps on each branch $P_{1}$ and $P_{2}$. The rule applies when at least one step is possible on each branch, and in the context of both standard and concurrent execution (which enables 'nested' concurrency).

The conclusion of the rule does two things. First, it merges the independent and 'local' updates to (copies of) belief base $\mathcal{B}$ by branches $P_{1}$ and $P_{2}$. We define $\operatorname{MergE}\left(\mathcal{B}_{1}, \mathcal{B}_{2}, \mathcal{B}\right)=$ $\left(\mathcal{B} \cup \mathcal{B}_{1}^{+} \cup \mathcal{B}_{2}^{+}\right) \backslash\left(\mathcal{B}_{1}^{-} \cup \mathcal{B}_{2}^{-}\right)$, where for both $i \in[1,2], \mathcal{B}_{i}^{+}=$ $\mathcal{B}_{i} \backslash \mathcal{B}$ and $\mathcal{B}_{i}^{-}=\mathcal{B} \backslash \mathcal{B}_{i}$. Second, the conclusion combines the action histories (if any) yielded by the two branches to form a new history, which is appended to $\mathcal{A}$. This notion of combining and appending is defined as follows. Let $\mathcal{A}, \mathcal{A}_{1}$, and $\mathcal{A}_{2}$ be as in rule $\|$. For both $i \in[1,2]$, let $\mathcal{A}_{i}=\mathcal{A} ; \mathcal{A}_{i}^{\prime}$ for some $\mathcal{A}_{i}^{\prime}$, or let $\mathcal{A}_{i}=\mathcal{A}$, i.e., no actions were yielded by the corresponding branch. Then, $\operatorname{APPEND}\left(\mathcal{A}_{1}, \mathcal{A}_{2}, \mathcal{A}\right)$ is defined as (i) $\mathcal{A} ;\left(\mathcal{A}_{1}^{\prime} \| \mathcal{A}_{2}^{\prime}\right)$ if $\mathcal{A}_{1}, \mathcal{A}_{2} \neq \mathcal{A}$, (ii) $\mathcal{A}_{2}$ if $\mathcal{A}_{1}=\mathcal{A}$, and (iii) $\mathcal{A}_{1}$ if $\mathcal{A}_{2}=\mathcal{A}$.

Rules $S e q_{1}^{\|}, S e q_{2}^{\|}$and $S e q_{3}^{\|}$give semantics for sequential execution in the context of concurrency. Rule $S e q_{1}^{\|}$applies when at least two execution steps are possible on a given sequential program $P_{1} ; P_{2}$. The antecedent executes one step on $P_{1}$, and then recursively performs all possible execution steps on the remainder $P_{1}^{\prime} ; P_{2}$. Rule $S e q_{3}^{\|}$applies when a given sequential program $P_{1} ; P_{2}$ (possibly a remainder) can terminate in one step. Rule $S e q_{2}^{\|}$is analogous to $S e q_{2}$, and applies when $P_{1}$ has finished and at least one step is possible on $P_{2}$. Formally, given the set of all programs $P_{\text {all }}$, function FIN : $P_{\text {all }} \mapsto\{\mathrm{T}, \perp\}$ indicates whether a given program $P \in P_{\text {all }}$ has finished. The function is defined as follows:

$$
\operatorname{FIN}(P)= \begin{cases}\operatorname{FIN}\left(P_{1}\right) \wedge \operatorname{FIN}\left(P_{2}\right) & \text { if } P \in\left\{P_{1} \nVdash P_{2}, P_{1} \mid P_{2}\right\}, \\ \operatorname{FIN}\left(P_{1}\right) & \text { if } P=P_{1} \triangleright P_{2}, \\ \top & \text { if } P=\eta, \\ \perp & \text { otherwise. }\end{cases}
$$

Proposition 1. If $\mathrm{FIN}(P)$ holds for some program $P$, then for any belief base $\mathcal{B}$ and action history $\mathcal{A}$, there is no $\mathcal{B}^{\prime}, \mathcal{A}^{\prime}$ and $P^{\prime}$ such that $[\mathcal{B}, \mathcal{A}, P] \stackrel{\mathrm{ONE}}{\longrightarrow}\left[\mathcal{B}^{\prime}, \mathcal{A}^{\prime}, P^{\prime}\right]$.

Proof. The case $P \in\left\{\eta, P_{1} \nVdash P_{2}\right\}$ (for some $P_{1}$ and $P_{2}$ ) is trivial as no rules can be applied to $P$. If $P=P_{1} \triangleright P_{2}$, we show that neither rule $\nabla_{\text {step }}$ nor $\triangleright_{\text {fail }}$ applies to $P$. Since $\operatorname{FIN}(P)$ holds, so does FIN $\left(P_{1}\right)$. Thus $\triangleright_{\text {fail }}$ cannot be applied to $P$. Similarly, $\nabla_{\text {step }}$ cannot be applied if $P_{1} \in$ $\left\{\eta, P_{1}^{1} \nVdash P_{1}^{2}\right\}$. If $P_{1}=P_{1}^{1} \triangleright P_{1}^{2}$, i.e., construct $\triangleright$ is 'nested' in $P$, the proposition follows because $\operatorname{FIN}\left(P_{1}^{1}\right)$ holds, and $\triangleright$ can only be nested to a finite depth in $P$. The cases where $P_{1}$ and $P$ are interleaved programs are proved similarly.

Finally, given an event-goal or interleaved program, rule Rewritell 'rewrites' it to make it the first program of a simple sequence, which is executed as above. This avoids the need for rules to handle programs $P_{1} \mid P_{2}$ and !e (and evolutions such as $P \triangleright P^{\prime}$ ) in the context of concurrency.

\subsection{An Example}

We will now illustrate some of the derivation rules in fig. 1 with an example. Consider a manufacturing facility with a robotic station that engraves the surfaces of wooden spheres. The station comprises a camera, a fixture that holds and rotates a sphere while other operations are being performed on it, and a robotic arm with a built-in circular tool changing rack comprising 6 tools (e.g. for milling and drilling).

The fixture can perform action $r X(N)$ (resp. $r Z(M)$ ), which rotates a wooden sphere, if it is currently in the fixture, $N$ (resp. $M$ ) times on the x-axis (resp. z-axis); each complete rotation takes 5 seconds and starts instantly. Both actions have precondition in, which is a proposition that holds only if a sphere is sensed in the fixture. In our scenario, $M=N=2$; the extra rotation on each axis leaves sufficient time for a concurrent preparatory (tool changing) action to complete. The camera performs action $r(N)$, which records, for $N$ seconds, a video of all the actions that are being performed on the sphere in the fixture; we use $N=25$.

The capabilities of the robot are as follows. Event-goal $e$, if it uses plan-rule $e:$ in $\wedge \neg a t \leftarrow c ; m(20)$, first prepares to engrave the sphere by changing the current tool to the milling tool, and then mills the sphere. Event-goal $e$ uses rule $e:$ in $\wedge$ at $\leftarrow m(20)$ if $i n$ holds and the milling tool was used last, i.e., proposition at holds. Action $c$ above rotates the tool rack until the milling tool is reached; it takes a second to rotate to the next tool on the rack (and thus at most 5 seconds). Action $m(N)$, with precondition $i n$, mills for $N$ seconds, which includes starting high speed rotation for the milling tool, moving it into the sphere, moving it out, and ending tool rotation, each of which takes negligible time. ${ }^{4}$

The derivation rule in the top row (eq. 1) of fig. 2 depicts one execution step on the concurrent program $r(25) \|$ !e $\|$ $(r X(2) \mid r Z(2))$, which specifies that while the sphere is being engraved, it should be (simultaneously) rotated on the $\mathrm{x}$-axis and the $\mathrm{z}$-axis (which can be performed in either order), and that all these activities should be recorded. The program is executed using rule $\|$, whose antecedent prescribes the concurrent execution of two programs, each using a step of type ALL. One such step is performed on action $r(25)$, and

\footnotetext{
${ }^{4}$ For a given axis of rotation, we assume there will be no difference in the engraving on the sphere whether milling is performed for exactly one rotation of the sphere or for longer (e.g. milling starts before the rotation, or ends after).
} 


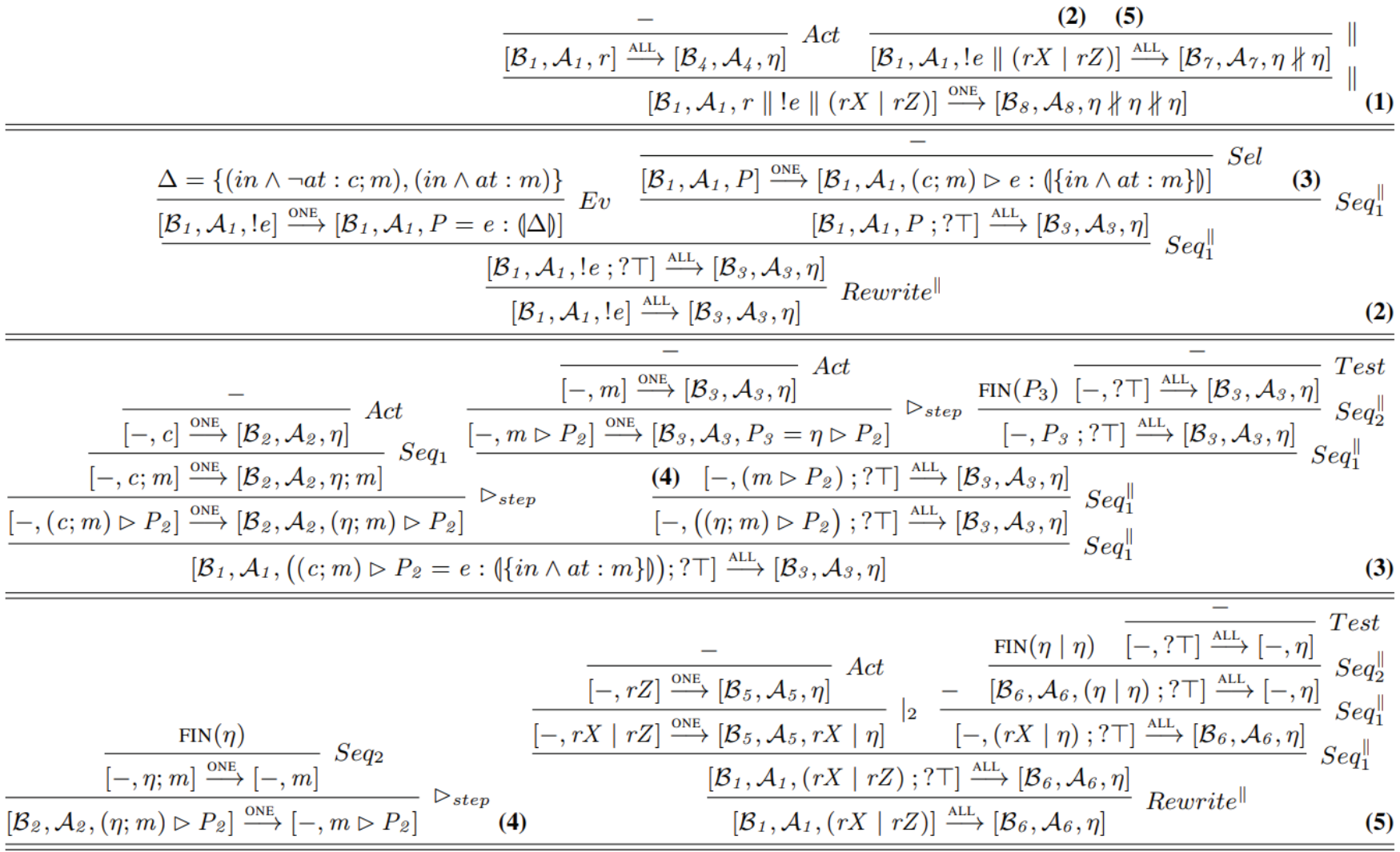

Figure 2: Equation 1 (first row) shows one execution step on concurrent program $r\|!\|(r X \mid r Z)$, which involves one step on $r$, and all the possible steps on both !e (eqs. 2-4 in rows 2-4) and $(r X \mid r Z)$ (eq. 5 in row 4). Action parameters are omitted, as are obvious antecedents, and obvious belief bases and action histories in configurations. The action histories above are: (i) $\mathcal{A}_{4}=\mathcal{A}_{1} ; r$, (ii) $\mathcal{A}_{2}=\mathcal{A}_{1} ; c$, (iii) $\mathcal{A}_{3}=\mathcal{A}_{2} ; m$, (iv) $\mathcal{A}_{5}=\mathcal{A}_{1} ; r Z$, (v) $\mathcal{A}_{6}=\mathcal{A}_{5} ; r X$, (vi) $\mathcal{A}_{7}=\mathcal{A}_{1} ;((c ; m) \|(r Z ; r X))$, and (vii) $\mathcal{A}_{8}=\mathcal{A}_{1} ;(r\|(c ; m)\|(r Z ; r X))$.

the other on concurrent 'subprogram' !e $\|(r X(2) \mid r Z(2))$ by recursively applying rule $\|$, whose antecedent, in turn, prescribes the concurrent execution of event-goal program !e (eq. 2) and interleaved program $r X(2) \mid r Z(2)$ (eq. 5).

Executing the top-level concurrent program yields action history $r(25)\|(c ; m(20))\|(r Z(2) ; r X(2))$, which $(i)$ abstractly represents all the associated action schedules (e.g. where the branches are interleaved, and where $r(25), c$, and $r Z(2)$ start together and overlap), ${ }^{5}$ and (ii) corresponds to the 'terminated branches' of the concurrent program.

\section{Multiple-Intention CCAN Semantics}

We now give our semantics for configurations with multiple intentions, i.e., agent configurations, which are of the form $[\Pi, \Lambda, \mathcal{B}, \mathcal{A}, \Gamma]$, where $\Gamma$ is a set of programs.

A transition between agent configurations is either of type CCAN, EVENT, or INT, and the transition relation on agent configurations is defined by the derivation rules in fig. 3 . Rule $A_{\text {ccan }}$ is the main rule, which represents the CCAN deliberation cycle. The CCAN-type step in the conclusion of

\footnotetext{
${ }^{5}$ Since, in systems such as PRS and JACK, branches ('threads') typically start execution at roughly the same point in time, we assume the same when writing concurrent branches in CCAN.
}

the rule involves two internal steps. In the first internal step, an intention is either $(i)$ removed (using rule $A_{\text {rem }}$ ) if it has failed or finished, (ii) progressed (using rule $A_{\text {int }}$ ) by one step, or (iii) progressed by multiple steps, in which case a concurrent program will have been executed. In the second internal step, newly observed event-goals from the (external) environment are processed (using rule $A_{e v}$ ), by creating an intention for each such event-goal.

\section{Properties of CCAN}

We now show that our concurrency semantics has three key properties: a concurrent program does not terminate 'prematurely' (before all the branches terminate), as ensured by JACK's fourth definition (sec. 1); the semantics is sound and complete in terms of the action histories that are produced; and if the concurrent program's branches are interleaved, any resulting action history corresponds to a valid action schedule for the concurrent program, i.e., the history is an ordering of a pomset yielded by the concurrent program. We use action histories because we are interested in exploring behavioural equivalence-we thus abstract from things such as belief bases, and 'unobservable steps' such as the creation of a relevant plan set (rule $E v$ in fig. 1). 


$$
\begin{aligned}
& \frac{[\mathcal{B}, \mathcal{A}, \Gamma] \stackrel{\text { INT }}{\Longrightarrow}\left[\mathcal{B}^{\prime}, \mathcal{A}^{\prime}, \Gamma^{\prime}\right]\left[\mathcal{B}^{\prime}, \mathcal{A}^{\prime}, \Gamma^{\prime}\right] \stackrel{\text { EVENT }}{\Longrightarrow}\left[\mathcal{B}^{\prime}, \mathcal{A}^{\prime}, \Gamma^{\prime \prime}\right]}{[\mathcal{B}, \mathcal{A}, \Gamma] \stackrel{\text { CCAN }}{\Longrightarrow}\left[\mathcal{B}^{\prime}, \mathcal{A}^{\prime}, \Gamma^{\prime \prime}\right]} A_{c c a n} \frac{P \in \Gamma \quad[\mathcal{B}, \mathcal{A}, P] \stackrel{\text { ONE }}{\longrightarrow}\left[\mathcal{B}^{\prime}, \mathcal{A}^{\prime}, P^{\prime}\right]}{[\mathcal{B}, \mathcal{A}, \Gamma] \stackrel{\text { INT }}{\Longrightarrow}\left[\mathcal{B}^{\prime}, \mathcal{A}^{\prime},(\Gamma \backslash\{P\}) \cup\left\{P^{\prime}\right\}\right]} A_{\text {int }}
\end{aligned}
$$

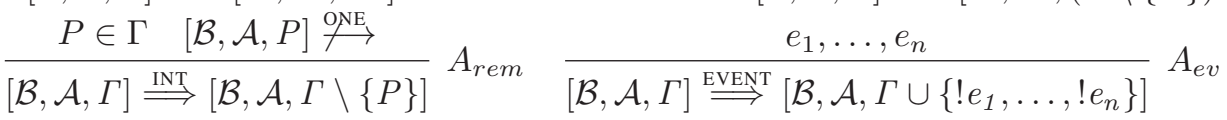

Figure 3: CCAN derivation rules for configurations with multiple intentions.

We first define the notion of an execution trace, which is a sequence of configurations obtained by performing ONE type execution steps. In the sequel, we assume that all $+b$ and $-b$ programs occurring in the plan-library $\Pi$ have been replaced by equivalent actions, and given a configuration $C=[\mathcal{B}, \mathcal{A}, P]$, we define $C_{\mathcal{B}}=\mathcal{B}, C_{\mathcal{A}}=\mathcal{A}$, and $C_{P}=P$.

Definition 1. An execution trace of a configuration $C=$ $[\mathcal{B}, \mathcal{A}, P]$ is a finite sequence of configurations $C_{1} \cdot \ldots \cdot C_{n}$ such that $C=C_{1}, n>1$, and $C_{i} \stackrel{\mathrm{ONE}}{\longrightarrow} C_{i+1}$ for all $i \in$ $[1, n-1]$; the trace is said to have terminated if $C_{n} \stackrel{\text { ONE }}{\longrightarrow}$.

The first theorem states that after one execution step on a concurrent program (which yields a terminated concurrent program $\left.P_{1}^{\prime} \nVdash \ldots \nVdash P_{n}^{\prime}\right)$, each branch will have performed all the possible (internal) execution steps and terminated.

Theorem 1. Let $\mathcal{B}$ be a belief base, $\mathcal{A}$ an action history, and $P_{\|}=P_{1}\|\ldots\| P_{n}$ a concurrent program s.t. $P_{i} \neq$ $P_{i}^{1} \| P_{i}^{2}$ (for any $P_{i}^{1}, P_{i}^{2}$ and $\left.i \in[1, n]\right){ }^{6}$ If $\left[\mathcal{B}, \mathcal{A}, P_{\|}\right] \stackrel{\text { ONE }}{\longrightarrow}$ $\left[\mathcal{B}^{\prime}, \mathcal{A}^{\prime}, P_{1}^{\prime} \nVdash \ldots \nVdash P_{n}^{\prime}\right]$, then $\left[\mathcal{B}^{\prime}, \mathcal{A}^{\prime}, P_{i}^{\prime}\right] \stackrel{\text { ONE }}{\longrightarrow}$ for $i \in[1, n]$.

Proof Sketch. Consider any $P_{i}$ above. From the assumption of the theorem and derivation rule $\|$, it follows that $\left[\mathcal{B}, \mathcal{A}, P_{i}\right] \stackrel{\text { ALL }}{\longrightarrow}\left[\mathcal{B}^{\prime \prime}, \mathcal{A}^{\prime \prime}, P_{i}^{\prime}\right]$, where $\mathcal{B}^{\prime \prime}$ and $\mathcal{A}^{\prime \prime}$ correspond to $P_{i}^{\prime}$ and form part of respectively $\mathcal{B}^{\prime}$ and $\mathcal{A}^{\prime}$.

If $P_{i} \in\{? \phi, a\}$, only rule Test or Act can apply, which guarantee that $\left[\mathcal{B}^{\prime \prime}, \mathcal{A}^{\prime \prime}, P_{i}^{\prime}\right] \stackrel{\text { NNE }}{\longrightarrow}$. If $P_{i} \in\left\{! e, P \mid P^{\prime}\right\}$ (for some $P$ and $P^{\prime}$ ), it is rewritten as a sequential program. Thus, the final case is where $P_{i}$ is a sequence, or evolves into one. Consider the former (the latter is analogous). Since rule $S e q_{2}^{\|}$cannot apply to configuration $\left[\mathcal{B}, \mathcal{A}, P_{i}\right]$ (because $P_{i}$ is a plan-body and thus unfinished), either rule $S e q_{1}^{\|}$or $\mathrm{Seq}_{3}^{\|}$must have been applied to $\left[\mathcal{B}, \mathcal{A}, P_{i}\right]$, both of whose antecedents ensure that $\left[\mathcal{B}^{\prime \prime}, \mathcal{A}^{\prime \prime}, P_{i}^{\prime}\right] \stackrel{\text { AlL }}{\longrightarrow}$ holds. Finally, we prove by contradiction that $\left[\mathcal{B}^{\prime \prime}, \mathcal{A}^{\prime \prime}, P_{i}^{\prime}\right] \stackrel{\text { NNE }}{\longrightarrow}$ also holds.

Let us assume instead that $\left[\mathcal{B}^{\prime \prime}, \mathcal{A}^{\prime \prime}, P_{i}^{\prime}\right] \stackrel{\text { ONE }}{\longrightarrow}$. Consider the case where $P_{i}^{\prime}=P_{i}^{1} ; P_{i}^{2}$ is a sequence such that $P_{i}^{1}$ is not a sequence and $\neg \operatorname{FIN}\left(P_{i}^{1}\right)$ holds. Since $\left[\mathcal{B}^{\prime \prime}, \mathcal{A}^{\prime \prime}, P_{i}^{1}\right] \stackrel{\mathrm{ONE}}{\longrightarrow}$ is entailed by our assumption, either the antecedent of rule $S e q_{1}^{\|}$or $S e q_{3}^{\|}$must hold w.r.t. $P_{i}^{\prime}$. This contradicts the fact that $\left[\mathcal{B}^{\prime \prime}, \mathcal{A}^{\prime \prime}, P_{i}^{\prime}\right] \stackrel{\text { AlL }}{\longrightarrow}$. The cases where $\operatorname{FIN}\left(P_{i}^{1}\right)$ holds or $P_{i}^{\prime}$ is not a sequence also lead to contradictions.

The theorem can be straightforwardly extended to show that, due to the definition of 'FIN' (sec. 3.2), the concurrent

\footnotetext{
${ }^{6}$ Any concurrent program can be represented in this 'full' form.
}

program $P_{\|}$succeeds, i.e., yields a terminated (concurrent) program that has finished, iff each branch $P_{i}$ has succeeded.

Theorem 2 concerns soundness and completeness for the derivation rules that relate to concurrent programs. Soundness is due to the fact that any action history yielded by a branch of a concurrent program can also be yielded when the branch is executed separately from the program (perhaps using only rules in the top half of fig. 1). Conversely, completeness is due to the fact that any action history yielded by a non-concurrent program upon its termination can also be yielded when the latter is a branch of a concurrent program.

Theorem 2. Let $\mathcal{B}, \mathcal{A}$ and $P_{\|}=P_{1}\|\ldots\| P_{n}$ be as above. There exists a transition $\left[\mathcal{B}, \mathcal{A}, P_{\|}\right] \stackrel{\mathrm{ONE}}{\longrightarrow}\left[\mathcal{B}^{\prime}, \mathcal{A} ; \mathcal{A}^{\prime}, P_{\|}^{\prime}\right]$, with $\mathcal{A}^{\prime}=\mathcal{A}_{1}\|\ldots\| \mathcal{A}_{n}$, iff there exists a terminated execution trace $C_{1}=\left[\mathcal{B}, \mathcal{A}, P_{i}\right] \cdot \ldots \cdot C_{m}=\left[\mathcal{B}^{\prime \prime}, \mathcal{A} ; \mathcal{A}_{i}^{\prime}, P_{i}^{\prime}\right]$ for each $i \in[1, n]$, such that $\mathcal{A}_{i}^{\prime}=\mathcal{A}_{i}{ }^{7}$

Proof Sketch. We discuss one direction of the proof: consider an execution trace $C_{1} \cdot \ldots \cdot C_{m}$ as above. The proof is involved, requiring induction on the length of the trace and the structure of each $C_{i}$. The main part is the inductive case, which takes any pair of configurations $C_{j}$ and $C_{j+1}$ (for $j \in[1, m-1]$ ), and the corresponding action history $\mathcal{A}^{\prime \prime}$ (if $\left.C_{j+1}\right|_{\mathcal{A}}=\left.C_{j}\right|_{\mathcal{A}} ; \mathcal{A}^{\prime \prime}$ ), and shows that $\mathcal{A}^{\prime \prime}$ can also be yielded by an ALL-type transition from $C_{j}$.

Let us consider the two main cases. In the first, $\left.C_{j}\right|_{P}=$ $P_{j}^{1} ; P_{j}^{2}$ (for some $P_{j}^{1}$ and $P_{j}^{2}$ ), and $P_{j}^{1}$ is not a sequential program and $\neg \mathrm{FIN}\left(P_{j}^{1}\right)$ holds. Since the transition $C_{j} \stackrel{\text { ONE }}{\longrightarrow}$ $C_{j+1}$ must have used rule $S e q_{1}$, it follows that transition $\left[\left.C_{j}\right|_{\mathcal{B}},\left.C_{j}\right|_{\mathcal{A}}, P_{j}^{1}\right] \stackrel{\mathrm{ONE}}{\longrightarrow}\left[\left.C_{j+1}\right|_{\mathcal{B}},\left.C_{j+1}\right|_{\mathcal{A}}, P_{j}^{1^{\prime}}\right]$ is possible, with $\left.C_{j+1}\right|_{P}=P_{j}^{1^{\prime}} ; P_{j}^{2}$. Thus, the first condition in the antecedents of both $S e q_{1}^{\|}$and $S e q_{3}^{\|}$holds. The interesting subcase is where $\neg \operatorname{FIN}\left(P_{j}^{1^{\prime}}\right)$. Now if $C_{j+1} \stackrel{\mathrm{ONE}}{\longrightarrow}$ (i.e., $j+1=m$ ), it follows that the second condition also holds in the antecedent of $S e q_{3}^{\|}$. Thus, $\mathcal{A}^{\prime \prime}$ can be yielded by applying the rule to $C_{j}$. If $C_{j+1} \stackrel{\text { ONE }}{\longrightarrow}$, the second condition holds in the antecedent of $S e q_{1}^{\|}$, and consequently also the third. Thus, $\mathcal{A}^{\prime \prime}$ can be yielded by applying the rule to $C_{j}$.

The second main case is where $\left.C_{j}\right|_{P}=! e$ (the case where $\left.C_{j}\right|_{P}=P_{j}^{1} \mid P_{j}^{2}$ is analogous). Then, transition $C_{j} \stackrel{\text { ONE }}{\longrightarrow}$ $C_{j+1}$ must have used rule $E v$, and $\left.C_{j+1}\right|_{P}=e:(\Delta)$. This transition can be simulated by applying rule Rewrite ${ }^{\|}$to $C_{j}$ : the rule's antecedent holds because we showed above

\footnotetext{
${ }^{7}$ We omit the trivial corollary where some $\mathcal{A}_{i}$ are 'empty'.
} 
that either $S e q_{1}^{\|}$or $S e q_{3}^{\|}$must be applicable to configurations such as $\left[\left.C_{j}\right|_{\mathcal{B}},\left.C_{j}\right|_{\mathcal{A}}, ! e ; ? \top\right]$.

The third theorem links concurrency and interleaving, and is based on the standard notions of a pomset and a linear extension of one. We first give some auxiliary definitions to represent action histories produced by a concurrent program in terms of pomsets, which enables comparing their linear extensions with the action histories that are produced when the program's branches are interleaved.

Definition 2. An action pomset (or simply a pomset) is a 4-tuple $\rho=\langle V, A, \prec, f\rangle$, where the 'vertices' $V$ is a finite set of natural numbers, $A$ is a finite set of actions, the 'ordering relation' $\prec$ is an irreflexive, a transitive, and an asymmetric binary relation on $V$, and the 'labelling function' $f$ is a surjection from $V$ to $A$. A sequence of actions $a_{1} ; \ldots ; a_{n}$ is a linear extension of a pomset $\rho$, denoted $a_{1} ; \ldots ; a_{n} \in \operatorname{LIN}(\rho)$, iff there exists a permutation $v_{1} \cdot \ldots \cdot v_{n}$ of the vertices of $\rho$ such that $\left(v_{i}, v_{j}\right) \in \prec$ implies $i<j$, and $a_{k}=f\left(v_{k}\right)$ for all $k \in[1, n] .^{8}$

Next, we define how a pomset is built from a given action history, by the recursive application of sequential and/or parallel composition operators. Let $\rho_{1}=\left\langle V_{1}, A_{1}, \prec_{1}, f_{1}\right\rangle$ and $\rho_{2}=\left\langle V_{2}, A_{2}, \prec_{2}, f_{2}\right\rangle$ be pomsets, and let $r$ (a 'renaming') be a bijection from $V_{2}$ to a set $V_{3}$ such that $V_{3} \cap V_{1}=\emptyset$ and $V_{3} \cap V_{2}=\emptyset$. Let $\left\langle V_{3}, A_{3}, \prec_{3}, f_{3}\right\rangle$ be the pomset obtained from $\rho_{2}$ by renaming it using $r$, i.e., replacing each occurrence in $\rho_{2}$ of each vertex $v \in V_{2}$ with $r(v)$. Then, we define $\rho_{1} \prec^{*} \rho_{2}$ as the pomset $\langle V, A, \prec, f\rangle$, where $V=V_{1} \cup V_{3}$, $A=A_{1} \cup A_{3}, f=f_{1} \cup f_{3}$, and $\prec=\prec_{1} \cup \prec_{3} \cup V_{1} \times V_{3}$; similarly, we define $\rho_{1} \cup^{*} \rho_{2}$ as the pomset $\left\langle V, A, \prec_{1} \cup \prec_{3}, f\right\rangle$. Finally, given an action history $\mathcal{A}$, we define the corresponding pomset as

$$
\mathbf{P}(\mathcal{A})= \begin{cases}\mathbf{P}\left(\mathcal{A}_{1}\right) \prec^{*} \mathbf{P}\left(\mathcal{A}_{2}\right) & \text { if } \mathcal{A}=\mathcal{A}_{1} ; \mathcal{A}_{2}, \\ \mathbf{P}\left(\mathcal{A}_{1}\right) \cup^{*} \mathbf{P}\left(\mathcal{A}_{2}\right) & \text { if } \mathcal{A}=\mathcal{A}_{1} \| \mathcal{A}_{2}, \\ \langle\{1\},\{a\}, \emptyset,\{(1, a)\}\rangle & \text { if } \mathcal{A}=a .\end{cases}
$$

Proposition 2. If $\mathcal{A}$ is an action history, $\boldsymbol{P}(\mathcal{A})$ is a pomset.

Proof. Let $a$ be any action. First, if action history $\mathcal{A}=a$, then $\mathbf{P}(\mathcal{A})$ is the 'atomic' pomset comprising the single action ' $a$ ', the empty ordering relation, and the single vertex ' 1 ' labelled with the action. Second, each incremental application of either $\prec^{*}$ or $\cup^{*}$ to two (initially 'atomic') pomsets $\rho_{1}$ and $\rho_{2}$ will also yield a pomset because $(i)$ the vertices in $\rho_{2}$ will be renamed to $V_{3}$ (guaranteeing that the resulting labelling function is a surjection), and (ii) the entire cross product of $V_{1}$ and $V_{3}$ is added to the resulting ordering relation in the definition of $\rho_{1} \prec^{*} \rho_{2}$, ensuring transitivity.

Finally, Theorem 3 states that if the branches of a concurrent program are executed as part of an interleaved program, any linear extension of (the pomset of) a resulting action history will also be a linear extension of some action history that is yielded by the concurrent program. We need to use linear extensions of the former action history (as opposed to using the history directly) to account for concurrent programs that might emerge within the branches as they evolve.

\footnotetext{
${ }^{8}$ We treat relations and functions as sets of ordered pairs.
}

Theorem 3. Let $\mathcal{B}, \mathcal{A}$ and $P_{\|}=P_{1}\|\ldots\| P_{n}$ be as above, and suppose $\left[\mathcal{B}, \mathcal{A}, P_{\|}\right] \stackrel{\text { ONE }}{\longrightarrow}$. Let $\left[\mathcal{B}, \mathcal{A}, P_{1}|\ldots| P_{n}\right] \cdot \ldots$. $\left[\mathcal{B}^{\prime}, \mathcal{A} ; \mathcal{A}_{\mid}, P^{\prime}\right]$ be a terminated execution trace. Then, there exists a transition $\left[\mathcal{B}, \mathcal{A}, P_{\|}\right] \stackrel{\mathrm{ONE}}{\longrightarrow}\left[\mathcal{B}^{\prime}, \mathcal{A} ; \mathcal{A}_{\|}, P_{\|}^{\prime}\right]$ such that $\operatorname{LIN}\left(\boldsymbol{P}\left(\mathcal{A}_{\mid}\right)\right) \subseteq \operatorname{LIN}\left(\boldsymbol{P}\left(\mathcal{A}_{\|}\right)\right)$.

Proof Sketch. Consider the case $n=2$, i.e., $P_{\|}=P_{1} \| P_{2}$, and let $\mathcal{T}$ be the above trace. Let $\mathcal{A}^{\prime} \in \operatorname{LIN}\left(\mathbf{P}\left(\mathcal{A}_{\mid}\right)\right)$be a linear extension corresponding to $\mathcal{T}$. Consider the subcase where the first $x$ actions in $\mathcal{A}^{\prime}$ are yielded by $P_{1}$, and the next $y$ (but not $y+1$ ) by $P_{2}$, with $x, y>0$. Now consider the prefix of $\mathcal{T}$ that yields the first $x$ actions. The prefix is also an execution trace from $\left[\mathcal{B}, \mathcal{A}, P_{1}\right]$ if we remove program $P_{2}$ from the prefix, i.e., we replace program $\left.C\right|_{P}=P_{1}^{\prime} \mid P_{2}$ in each configuration $C$ in the prefix by $P_{1}^{\prime}$.

Suppose the prefix ends with $C^{1}=\left[\mathcal{B}^{1}, \mathcal{A}^{1}, P_{1}^{\prime} \mid P_{2}\right]$. Consider the trace $\mathcal{T}^{\prime}$ that starts from $C^{1}$ in $\mathcal{T}$, executes only $P_{2}$, and ends immediately on yielding the next $y$ actions. Suppose $\mathcal{T}^{\prime}$ ends with $C^{2}=\left[\mathcal{B}^{2}, \mathcal{A}^{2}, P_{1}^{\prime} \mid P_{2}^{\prime}\right]$. While belief bases $\mathcal{B}$ and $\mathcal{B}^{1}$ may differ, we can show that $\mathcal{T}^{\prime}$ can be simulated by a trace from $\left[\mathcal{B}, \mathcal{A}, P_{2}\right]$ that ends with a configuration $C$ s.t. $\left.C\right|_{P}=P_{2}^{\prime}$. This follows from our assumption in sec. 2 that $P_{1}$ and $P_{2}$ are unrelated. A similar reasoning can be applied to the next actions yielded by $P_{1}^{\prime}$ and $P_{2}^{\prime}$, by the resulting $P_{1}^{\prime \prime}$ and $P_{2}^{\prime \prime}$, and so on, until we build terminated execution traces for $\left[\mathcal{B}, \mathcal{A}, P_{1}\right]$ and $\left[\mathcal{B}, \mathcal{A}, P_{2}\right]$. Using the traces, we can show that the antecedent of rule $\|$ holds for $\left[\mathcal{B}, \mathcal{A}, P_{\|}\right]$, from which the theorem also follows.

The converse of the theorem does not hold due to our constraint in sec. 2 that no other (non-concurrent) step can be executed while a concurrent program is being executed. For example, take concurrent program $P=\left(\left(a_{1} \| a_{2}\right) ; a_{3}\right) \|$ $a_{4}$, which also represents its action history $\mathcal{A}$. While the sequence $a_{1} ; a_{4} ; a_{2} ; a_{3}$ is a linear extension of the pomset of $\mathcal{A}$, the sequence cannot be produced by the interleaved program corresponding to $P$, namely, $\left(\left(a_{1} \| a_{2}\right) ; a_{3}\right) \mid a_{4}$.

\section{Discussion}

We provide a BDI agent programming language supporting (true) concurrency. This differs from past work on similar languages, which interpret concurrency as interleaving. We support 'nested' concurrency, and a limited form of nesting between concurrency and interleaving. We prove key properties of the semantics in relation to concurrency, e.g. soundness and completeness w.r.t. action histories. Our results can be extended to develop further notions, e.g. what it means for a program to be 'more concurrent' than another.

Concurrency enables branches to be executed on separate processors or machines, yielding smaller makespans than would otherwise be possible. Concurrency also enables particular desirable action schedules (or expressing an aspect of 'user intent' (Fox 1997; Kambhampati, Mali, and Srivastava 1998)) that would not be possible by interleaving actions, e.g. the schedule in which the recording action $r(25)$ spans over both the rotate actions $r X(2)$ and $r Z(2)$.

There are many interesting avenues to explore in future work. In particular, we could formalise other approaches to concurrency, such as the more sophisticated approach of 
immediately aborting execution of the remaining branches upon the failure of one branch in a concurrent program (Harland et al. 2015). The semantics that we have presented is a crucial step toward formalising such alternative approaches, which would then enable formal comparison. We could also explore how to relax our constraint on how a concurrent program can be interleaved; enable interaction between concurrent branches, e.g. to communicate the binding assigned to a variable that is shared between multiple branches; add concurrency to other systems, e.g. AgentSpeak; model check concurrent (user supplied) JACK plans (cf. (Bordini et al. 2003)); and explore what concurrency means in the presence of advanced agent constructs, e.g. the declarative goal (e.g. (Hindriks et al. 2001; Winikoff et al. 2002)) and planning (e.g. (Sardina and Padgham 2011)) constructs.

\section{References}

Allen, J. F. 1983. Maintaining knowledge about temporal intervals. Communications of the ACM 26(11):832-843.

Bordini, R. H., and Hübner, J. F. 2010. Semantics for the Jason variant of AgentSpeak:(plan failure and some internal actions). In Proc. of the European Conf. on Artificial Intelligence (ECAI), 635640.

Bordini, R. H.; Fisher, M.; Pardavila, C.; and Wooldridge, M. 2003. Model Checking AgentSpeak. In Proc. of the International Conf. on Autonomous Agents and Multiagent Systems (AAMAS), 409416.

Boudol, G., and Castellani, I. 1987. On the semantics of concurrency: Partial orders and transition systems. In Proc. of the International Joint Conf. on Theory and Practice of Software Development (TAPSOFT), 123-137.

Boudol, G., and Castellani, I. 1988a. Concurrency and atomicity. Theoretical Computer Science 59(1):25-84.

Boudol, G., and Castellani, I. 1988b. A non-interleaving semantics for CCS based on proved transitions. Fundamenta Informaticae XI:433-453.

Boudol, G., and Castellani, I. 1989. Permutation of transitions: An event structure semantics for CCS and SCCS. In Linear Time, Branching Time and Partial Order in Logics and Models for Concurrency, 411-427. Springer Berlin Heidelberg.

Busetta, P.; Rönnquist, R.; Hodgson, A.; and Lucas, A. 1999. Jack intelligent agents - components for intelligent agents in Java. Technical report, Agent Oriented Software.

Chaouche, A. C.; Seghrouchni, A. E. F.; Ilié, J.-M.; and Saïdouni, D.-E. 2014. A dynamical plan revising for ambient systems. Procedia Computer Science 32:37-44.

Dastani, M. 2008. 2APL: A practical agent programming language. Autonomous Agents and Multi-Agent Systems (JAAMAS) 16(3):214-248.

de Giacomo, G.; Lespérance, Y.; and Levesque, H. J. 2000. ConGolog, a concurrent programming language based on the situation calculus. Artificial Intelligence 121(1-2):109-169.

de Silva, L.; Meneguzzi, F.; and Logan, B. 2018. An operational semantics for a fragment of PRS. In Proc. of the International Joint Conf. on Artificial Intelligence, (IJCAI), 195-202.

de Silva, L.; Sardina, S.; and Padgham, L. 2016. Summary information for reasoning about hierarchical plans. In Proc. of the European Conf. on Artificial Intelligence (ECAI), 1300-1308.
Degano, P.; De Nicola, R.; and Montanari, U. 1985. Partial ordering derivations for CCS. In Fundamentals of Computation Theory, 520-533. Springer Berlin Heidelberg.

Degano, P.; De Nicola, R.; and Montanari, U. 1990. A partial ordering semantics for CCS. Theoretical Computer Science 75(3): 223 262.

Fox, M. 1997. Natural hierarchical planning using operator decomposition. In Proc. of the European Conf. on Planning (ECP), 195-207.

Georgeff, M. P., and Ingrand, F. F. 1989. Decision-making in an embedded reasoning system. In Proc. of the International Joint Conf. on Artificial Intelligence (IJCAI), 972-978.

Harland, J.; Morley, D. N.; Thangarajah, J.; and Yorke-Smith, N. 2015. Aborting, suspending, and resuming goals and plans in BDI agents. Autonomous Agents and Multi-Agent Systems (JAAMAS) 31(2):288-331.

Hindriks, K. V.; de Boer, F. S.; van der Hoek, W.; and Meyer, J.J. C. 2001. Agent programming with declarative goals. In Proc. of Intelligent Agents VII. Agent Theories Architectures and Languages, Seventh International Workshop. Springer-Verlag. 228 243.

Huber, M. J. 1999. JAM: a BDI-theoretic mobile agent architecture. In Proc. of the Annual Conf. on Autonomous Agents, 236-243.

Kambhampati, S.; Mali, A.; and Srivastava, B. 1998. Hybrid planning for partially hierarchical domains. In Proc. of the National Conf. on Artificial Intelligence (AAAI), 882-888.

Morley, D., and Myers, K. 2004. The SPARK agent framework. In Proc. of the International Joint Conf. on Autonomous Agents and Multiagent Systems (AAMAS), 714-721.

Nunes, I.; Lucena, C.; and Luck, M. 2011. BDI4JADE: a BDI layer on top of JADE. In Proc. of the Workshop on Programming Multiagent Systems, 88-103.

Plotkin, G. D. 1981. A structural approach to operational semantics. Technical Report DAIMI FN-19, University of Aarhus, Denmark.

Rao, A. S. 1996. AgentSpeak(L): BDI agents speak out in a logical computable language. In Proc. of the European Workshop on Modelling Autonomous Agents in a Multi-Agent World, 42-55. Springer-Verlag.

Sardina, S., and Padgham, L. 2011. A BDI agent programming language with failure handling, declarative goals, and planning. $A u-$ tonomous Agents and Multi-Agent Systems (JAAMAS) 23(1):1870.

Sardina, S.; de Silva, L.; and Padgham, L. 2006. Hierarchical planning in BDI agent programming languages: A formal approach. In Proc. of the International Conf. on Autonomous Agents and Multiagent Systems (AAMAS), 1001-1008.

Winikoff, M.; Padgham, L.; Harland, J.; and Thangarajah, J. 2002. Declarative \& procedural goals in intelligent agent systems. In Proc. of the International Conf. on Principles of Knowledge Representation and Reasoning (KR), 470-481. 